\title{
The pion diffractive dissociation into two jets
}

\author{
V.L. Chernyak \\ Budker Institute for Nuclear Physics, \\ 630090 Novosibirsk, Russia \\ E-mail: chernyak@inp.nsk.su
}

\begin{abstract}
The method used and results obtained are described for calculation of the cross section for the pion diffractive dissociation into two jets. The main new qualitative result is that the distribution of longitudinal momenta for jets is not simply proportional to the profile of the pion wave function $\phi_{\pi}(x)$, but depends on it in a complicated way. In particular, it is shown that under conditions of the E791 experiment, the momentum distribution of jets is similar in its shape for the asymptotic and $\mathrm{CZ}$ wave functions. It is concluded therefore that, unfortunately, the process considered is really weakly sensitive to the profile of the pion wave function, and the accuracy of data is insufficient to distinguish clearly between different models of $\phi_{\pi}(x)$.

Comparison with the results of other papers on this subject is given.
\end{abstract}


1. The E791 experiment at Fermilab [1] has recently measured the cross section of the hard diffractive dissociation of the pion into two jets. In particular, the distribution of the total pion longitudinal momentum into fractions $y_{1}$ and $y_{2},\left(y_{1}+y_{2}\right)=1$, between jets has been measured. The main purpose was to obtain in this way the information about the leading twist pion wave function $\phi_{\pi}\left(x_{1}, x_{2}\right)$, which describes the distribition of quarks inside the pion in the longitudinal momentum fractions $x_{1}$ and $x_{2}=1-x_{1}$.

The hope was based on theoretical calculations of this cross section, see [2]-[5]. It has been obtained in these papers that the cross section is simply proportional to the pion wave function squared: $d \sigma / d y_{1} \sim\left|\phi_{\pi}\left(y_{1}\right)\right|^{2}$. In such a case, it would be sufficient to measure only the gross features of $d \sigma / d y$ to reveal the main characteristic properties of $\phi_{\pi}(x)$, and to discriminate between various available models of $\phi_{\pi}(x)$.

Our main qualitative result [6]-[7] is that this is not the case. The real situation is much more complicated, with $d \sigma / d y$ depending on $\phi_{\pi}(x)$ in a highly nontrivial way. We describe below in a short form our approach and the results obtained for this cross section.

2. The kinematics of the process is shown in fig.1. We take the nucleon as a target, and the initial and final nucleons are substituted by two soft gluons with momenta $q_{1}=(u+\xi) \bar{P}$ and $q_{2}=(u-\xi) \bar{P}, \bar{P}=$ $\left(P+P^{\prime}\right) / 2, \Delta=\left(q_{1}-q_{2}\right)$. 1 The lower blob in fig.1 represents the generalized gluon distribution of the nucleon, $G_{\xi}(u)=G_{\xi}(-u)$ [8]-[10].

The final quarks are on shell, carry the fractions $y_{1}$ and $y_{2}$ of the initial pion momentum, and their transverse momenta are: $\left(\mathbf{k}_{\perp}+\left(\mathbf{q}_{\perp} / 2\right)\right)$ and $\left(-\mathbf{k}_{\perp}+\left(\mathbf{q}_{\perp} / 2\right)\right), q_{\perp} \ll k_{\perp}$, where $q_{\perp}$ is the small final transverse momentum of the target, while $k_{\perp}$ is large. The invariant mass of these two quark jets is: $M^{2}=k_{\perp}^{2} / y_{1} y_{2}$, and $\xi=M^{2} / \nu, \nu=(s-u)$.

The upper blob $\mathrm{M}$ in fig.1 represents the hard kernel of the amplitude. According to the well developed approach to description of hard exclusive processes in QCD [11]-[14] (see [15] for a review), all hard gluon and quark lines in all diagrams have to be written down explicitly and substituted by their perturbative propagators. In other words, the hard momentum flow have to be made completely explicit and these hard lines of diagrams constitute the hard kernel M. They should not be hidden (if it is possible at all) as (a derivatives of) "the tails" of the unintegrated pion wave function

1 The small skewedness, $\xi \ll 1$, is always implied. It is typically: $\xi \sim 10^{-2}$, in the Fermilab experiment. 
$\Psi_{\pi}\left(x, l_{\perp}\right)$, or of the "unintegrated gluon distribution". This is, first of all, what differs our approach from previous calculations of this process in [4]-[5].

For calculation of $\mathrm{M}$ in the leading twist approximation and in the lowest order in $\alpha_{s}$, the massless pion can be substituted by two massless on shell quarks with the collinear momenta $x_{1} p_{\pi}$ and $x_{2} p_{\pi}$ and with zero transverse momenta, as account of primordial virtualities and transverse momenta results only in higher twist corrections to $\mathrm{M}$. The leading twist pion wave function $\phi_{\pi}\left(x, \mu_{o}\right)$ describes the distribution of these quarks in momentum fractions $x_{1}$ and $x_{2}$. P

So, the hard kernel $\mathrm{M}$ is proportional to the scattering amplitude of two initial collinear and on shell quarks of the pion on the on shell gluon:

$$
M \sim\left\{d\left(x_{1} p_{\pi}\right)+\bar{u}\left(x_{2} p_{\pi}\right)+g\left(q_{1}\right) \rightarrow d\left(p_{1}\right)+\bar{u}\left(p_{2}\right)+g\left(q_{2}\right)\right\} .
$$

In lowest order in $\alpha_{s}\left(k_{\perp}\right) \quad \mathrm{M}$ consists of 31 connected Born diagrams, each one is $\sim O\left(\alpha_{s}^{2}\left(k_{\perp}\right)\right)$.

The general structure of the amplitude is, therefore (symbolically):

$$
T \sim\left\langle P^{\prime}\left|A^{\perp} \cdot A^{\perp}\right| P\right\rangle \otimes\left(\bar{\psi}_{1} M \psi_{2}\right) \otimes\left\langle 0|\bar{u} \cdot d| \pi^{-}\right\rangle,
$$

where the first matrix element introduces the skewed gluon distribution of the nucleon $G_{\xi}(u), \bar{\psi}_{1}$ and $\psi_{2}$ are the free spinors of final quarks, "M" is the hard kernel, i.e. the product of all vertices and hard propagators, the last matrix element introduces the pion wave function $\phi_{\pi}(x)$, and $\otimes$ means the appropriate convolution.

3. Proceeding in the above described way and summing up con-

\footnotetext{
${ }^{2}$ As usual, on account of leading logs from loops the soft pion wave function $\phi_{\pi}\left(x, \mu_{o}\right)$ evolves to $\phi_{\pi}(x, \mu)$, where $\mu$ is the characteristic scale of the process. In other words, in the leading twist component of the pion wave function the two pion quarks prepare themselves for a hard collision by exchanging gluons and increasing their virtualities and transverse momenta from the initial scale $\mu_{o} \sim \Lambda_{Q C D}$ up to the characteristic scale of the process $\mu \leq k_{\perp}$, so that this quark pair enters nucleon (nuclei) having already the small transverse size $r_{\perp} \sim 1 / k_{\perp}$ (while the smallness of the longitudinal size is ensured by the Lorenz contraction).
} 


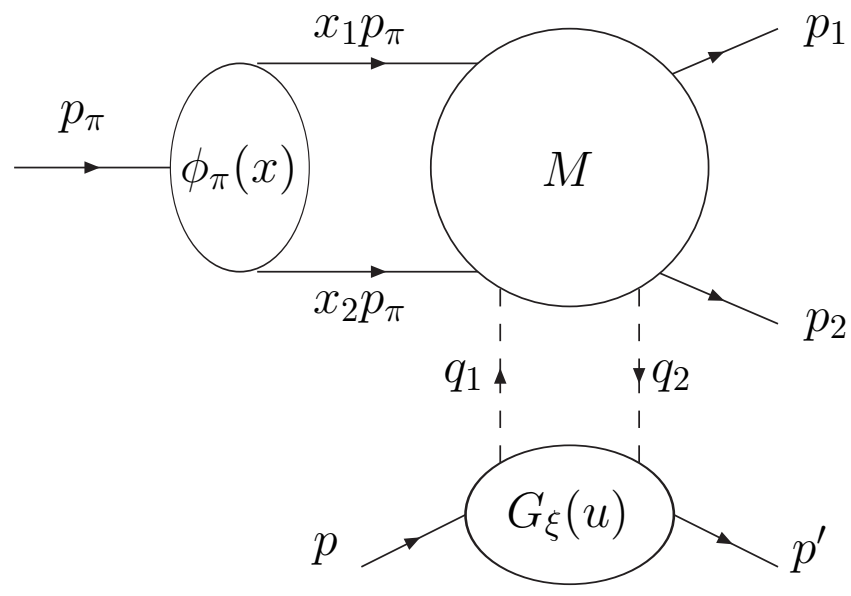

Figure 1: Kinematics and notations

tributions of all Born diagrams, one obtains for the cross section [ :

$$
\begin{gathered}
d \sigma_{N}=\frac{1}{8(2 \pi)^{5}} \frac{1}{s^{2}}|T|^{2} \frac{d y_{1}}{y_{1} y_{2}} d^{2} k_{\perp} d^{2} q_{\perp} ; \operatorname{Im} T_{g}=\frac{2 \pi s \omega_{o}}{k_{\perp}^{2}} G_{\xi}(\xi) \Omega, \\
\Omega=\int_{0}^{1} d x_{1} \phi_{\pi}\left(x_{1}\right)\left(\Sigma_{1}+\Sigma_{2}+\Sigma_{3}+\Sigma_{4}\right), \\
\Sigma_{1}=\left[\frac{4}{x_{1} x_{2}\left|x_{1}-y_{1}\right|} \frac{G_{\xi}(\bar{u})}{G_{\xi}(\xi)} \Theta\left(\left|x_{1}-y_{1}\right|>\delta\right)\right]+\left(y_{1} \leftrightarrow y_{2}\right), \\
\left.+\left[\left|x_{1}-y_{1}\right|\left(x_{1}-y_{2}\right)^{2} \frac{G_{\xi}(\bar{u})}{G_{\xi}(\xi)} \Theta\left(\left|x_{1}-y_{1}\right|>\delta\right)+\left(y_{1} \leftrightarrow y_{2}\right)\right]\right\},
\end{gathered}
$$

${ }^{3}$ Only the imaginary part of the amplitude, $\operatorname{Im} T_{g}$ (from the gluon distribution in the target), is shown explicitly in Eqs.(1)-(6), as it is expected to be the main one at high energies. For obtaining $\operatorname{Im} T$, the terms $i \epsilon$ were introduced into all denominators through: $s \rightarrow s+i \epsilon$, i.e. $\xi \rightarrow \xi-i \epsilon[6]$. The contribution of the quark distribution to $\operatorname{Im} T$ is given explicitly in [7]. Let us note also that all integrals over " $x_{1}$ " entering Eqs.(2)-(6) are convergent at the ends points $x_{1} \rightarrow 0$ or $x_{1} \rightarrow 1$. 


$$
\begin{gathered}
\Sigma_{3}=\frac{x_{1} x_{2}+y_{1} y_{2}}{9 x_{1}^{2} x_{2}^{2} y_{1} y_{2}}\left\{-1+\left[\left|x_{1}-y_{1}\right| \frac{G_{\xi}(\bar{u})}{G_{\xi}(\xi)} \Theta\left(\left|x_{1}-y_{1}\right|>\delta\right)+\left(y_{1} \leftrightarrow y_{2}\right)\right]\right\} \\
\Sigma_{4}=\frac{16}{9} \frac{1}{x_{1} x_{2} y_{1} y_{2}} \xi \frac{d G_{\xi}(u) /\left.d u\right|_{u=\xi}}{G_{\xi}(\xi)} ; \quad \delta=\frac{k_{\perp}^{2}}{s} \\
\omega_{o}=\frac{\delta_{i j}\left(4 \pi \alpha_{s}\right)^{2} f_{\pi}}{96}\left(\bar{\psi}_{1} \Delta_{\mu} \gamma_{\mu} \gamma_{5} \psi_{2}\right) \frac{\left(y_{1} y_{2}\right)^{2}}{k_{\perp}^{4}} ; \quad \bar{u}=\xi\left(\frac{x_{1} y_{2}+x_{2} y_{1}}{x_{1}-y_{1}}\right) .
\end{gathered}
$$

It is seen from the above equations that $d \sigma / d y$ is not $\sim\left|\phi_{\pi}(y)\right|^{2}$, but depends on the profile of $\phi_{\pi}(x)$ in a very complicated way.

4. In this section we present some numerical estimates of the cross section, based on the above expressions (1)-(6). Our main purpose was to trace the distribution of jets in longitudinal momentum fractions $y_{1}, y_{2}$ depending on the profile of the pion wave function $\phi_{\pi}(x)$.

a) For the skewed gluon distribution $G_{\xi}(u, t, \mu)$ of the nucleon at $t \simeq$ $-q_{\perp}^{2} \simeq 0$ we used the simple form (as we need it at $|u| \geq \xi$ only, and because $G_{\xi}(u) \rightarrow G_{o}(u)$ at $\left.|u| \gg \xi\right):$

$$
\left.G_{\xi}\left(u, t=0, \mu \simeq k_{\perp} \simeq 2 G e V\right)\right|_{u \geq \xi} \simeq u^{-0.3}(1-u)^{5} .
$$

This form agrees numerically reasonably well with the ordinary, $G_{o}(u, \mu \simeq$ $2 \mathrm{GeV})$, and skewed, $G_{\xi}(u, t=0, \mu \simeq 2 \mathrm{GeV})$, gluon distributions of the nucleon calculated in [16] and [17] respectively (in the typical region of the E791 experiment: $|u| \geq \xi \sim 10^{-2}$ ).

c) As for the pion leading twist wave function, $\phi_{\pi}(x, \mu)$, we compare two model forms: the asymptotic form, $\phi_{\pi}^{a s y}(x, \mu)=6 x_{1} x_{2}$, and the CZ-model [18]. The latter has the form: $\phi_{\pi}^{C Z}\left(x, \mu_{o} \simeq 0.5 \mathrm{GeV}\right)=30 x_{1} x_{2}\left(x_{1}-x_{2}\right)^{2}$, at the low normalization point. Being evolved to the characteristic scale of this process, $\mu \simeq k_{\perp} \simeq 2 \mathrm{GeV}$, it looks as: $\phi_{\pi}^{C Z}(x, \mu \simeq 2 G e V)=15 x_{1} x_{2}\left[\left(x_{1}-\right.\right.$ $\left.\left.x_{2}\right)^{2}+0.2\right]$, see Fig.2.

The results of these numerical calculations are then compared with the E791-data, see Fig.3. 


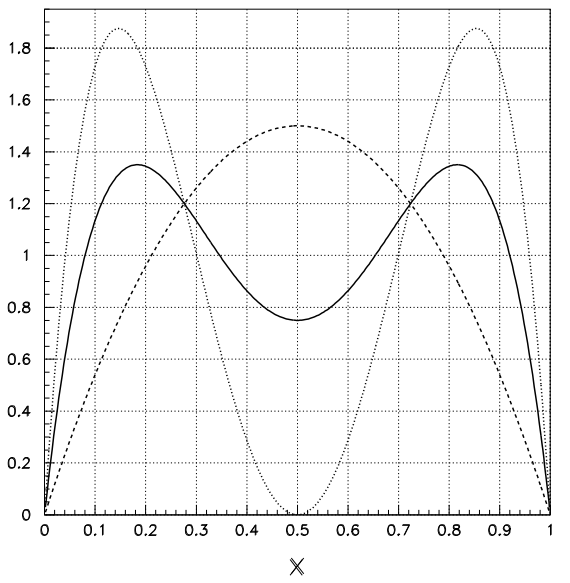

Figure 2: Profiles of the pion wave functions: a) $\phi_{\pi}^{C Z}(x, \mu \simeq 0.5 \mathrm{GeV})=$ $30 x_{1} x_{2}\left(x_{1}-x_{2}\right)^{2}$ - dotted line; b) $\phi_{\pi}^{C Z}(x, \mu \simeq 2 \mathrm{GeV})=15 x_{1} x_{2}\left[0.2+\left(x_{1}-x_{2}\right)^{2}\right]$ - solid line; c) $\phi_{\pi}^{a s y}(x)=6 x_{1} x_{2}$ - dashed line.

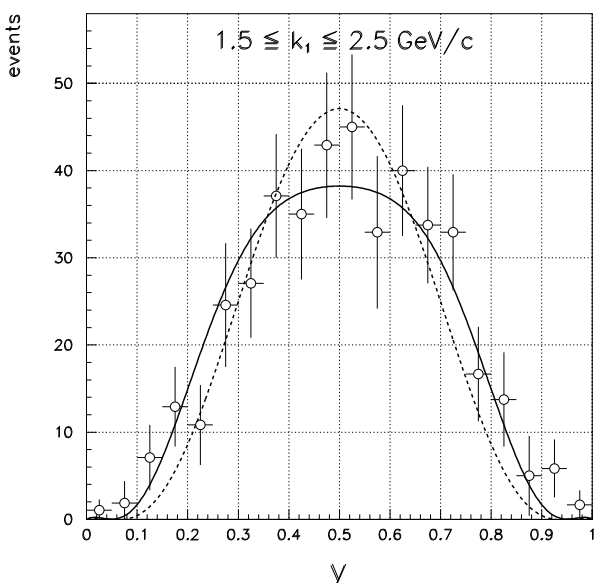

Figure 3: The y-distribution of jets calculated for $k_{\perp}=2 \mathrm{GeV}, E_{\pi}=500 \mathrm{GeV}$ and with the pion wave functions: $\phi_{\pi}^{C Z}(x, \mu \simeq 2 \mathrm{GeV})$ - solid line, $\phi_{\pi}^{\text {asy }}(x)$ - dashed line. The overall normalization is arbitrary, but the relative normalization of two curves is as calculated. The data points are from the E791 experiment [1]. 
It is seen that while the two model pion wave functions are quite different, the resulting distributions of jets in longitudinal momenta are similar and, it seems, the present experimental accuracy is insufficient to distinguish clearly between them. Moreover, even the ratio of the differential cross sections is not much different from unity: $d \sigma^{a s y} / d \sigma^{C Z} \simeq 1.2$ at $y_{1}=0.5$, and the same ratio is $\simeq 0.7$ at $y_{1}=0.25$. f

5. The purpose of this section is to compare (in a short form) the above results and those obtained by other authors, see [4], [5] and [19]-[20].

a) In comparison with Eqs.(1)-(6), the approximations used in [5] correspond to neglecting the terms $\Sigma_{2}, \Sigma_{3}$ and $\Sigma_{4}$, and supposing the region of small $\left|x_{1}-y_{1}\right|$ dominates the only remaining contribution: $\int d x_{1} \phi_{\pi}\left(x_{1}\right) \Sigma_{1} \simeq$ $\phi_{\pi}\left(y_{1}\right) \int d x_{1} \Sigma_{1}$. In this approximation, indeed, $\operatorname{Im} T_{g} \sim \phi_{\pi}(y)$ and $d \sigma / d y \sim$ $\left|\phi_{\pi}(y)\right|^{2}$.

Unfortunately, at the real conditions of the E791 experiment this is a poor approximation, and on account of neglected terms in Eqs.(1)-(6) the form of $d \sigma / d y$ changes qualitatively in comparison with $\left|\phi_{\pi}(y)\right|^{2}$, see Figs.2 and 3. p

b) The authors of papers in [4] argued that from the whole set of 31 Feynman diagrams only a small especially chosen subset dominates $\operatorname{Im} T_{g}$, while contributions of all other diagrams will be suppressed by Sudakov form factors. From this subset of diagrams they obtained also: $\operatorname{Im} T_{g}(y) \sim \phi_{\pi}(y)$.

First, I am unable to understand this last result, as the direct calculation of this subset of diagrams according to the rules described above in sect. 2 shows that, here also, resulting $\operatorname{Im} T_{g}(y)$ depends on $\phi_{\pi}(x)$ through a complicated integrated form, like those in Eqs.(4)-(6) above.

As for the Sudakov suppression of all other diagrams, I also disagree. The line of reasoning in [4] was the following. Consider, for instance, the diagrams like those in Fig.2 in [6]. Exchanging the hard gluon, the two pion quarks undergo abrupt change of their direction of motion and will tend to radiate

\footnotetext{
${ }^{4}$ On account of the quark contributions to $\operatorname{Im} T$ the resulting curves for $d \sigma / d y$ remain very similar to those in Fig.3, see [7]. For the numerical calculation of ReT see [20]. The estimate of $\operatorname{Re} T$ can be obtained from the well known formula: $\operatorname{Re} T(\nu) \simeq$ $(\pi / 2) d \ln \left(\nu^{-1} \operatorname{Im} T(\nu)\right) / d \ln \nu$. Because $\nu^{-1} \operatorname{Im} T(\nu) \sim \nu^{0.3}$ here, $\operatorname{Re} T \simeq 0.45 \operatorname{Im} T$ and $|T|^{2} \simeq 1.2(\operatorname{Im} T)^{2}$.

5 The forms of $d \sigma / d y$ for $\phi_{\pi}^{a s y}(x)$ and $\phi_{\pi}^{C Z}(x, \mu \simeq 2 \mathrm{GeV})$ and with the pion energy ten times larger, $E_{\pi}=5 \mathrm{TeV}, k_{\perp}=2 \mathrm{GeV}$, are shown in Fig.6 in [6]. It is seen from therein that even this does not help much (as the form of the distribution in $y$ of $d \sigma / d y$ weakly depends on the pion energy), and so the approximation used in [5] remains poor even at such energies.
} 
gluons in their previous direction of motion. Because the events with such radiation are excluded from the data set, what remains will be suppressed by the Sudakov form factors.

Applying this line of reasoning to nearly any hard exclusive amplitude, one will conclude that all such amplitudes will be suppressed, as a whole, by Sudakov effects. For instance, let us consider the large angle Compton amplitude. In the c.m.s. and at high energy, the three proton quarks change abruptly their direction of motion, deviating by large angle $\theta$. So, following the line of reasoning in [4], the whole large angle Compton amplitude will be suppressed by the Sudakov form factors.

Really, this is not the case. The reason is that the radiations of three collinear proton quarks cancel each other due to colour neutrality of the

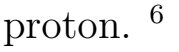

c) Unlike [4] and [5], the results from [19]-[20] are very similar to those from [6]-[7]. In particular, the analytic expressions for $\left(T_{g}+T_{q}\right)$ in terms of corresponding integrals over $\phi_{\pi}(x)$ and the gluon (quark) distributions are the same, the only difference is that the signs of $i \epsilon$ in denominators of a few terms are opposite. For $T_{g}$ for instance (and similarly for $T_{q}$ ), this difference can be represented as:

$$
\begin{gathered}
T_{g}=\delta T_{g}\left(\nu+i \epsilon, M^{2}\right)+\int_{0}^{1} d x_{1} \phi_{\pi}\left(x_{1}\right)\left[f_{1}(x, y) g^{+}\left(\nu, M^{2}\right)+f_{2}(x, y) g^{ \pm}\left(\nu, M^{2}\right)\right], \\
g^{ \pm}\left(\nu, M^{2}\right)=\int_{-1}^{1} d u \frac{G_{\xi}(u)}{\left(u+\frac{M^{2}}{\nu \pm i \epsilon}\right)\left(u-\frac{M^{2}}{\nu \pm i \epsilon}\right)}
\end{gathered}
$$

where $\delta T_{g}$ is the same in [6] and [19]-[20], and $f_{1}(x, y)$ and $f_{2}(x, y)$ are definite simple functions. The upper sign in Eq.(9) is from [6] (where as pointed out above, the terms $i \epsilon$ were introduced into all denominators through: $\nu \rightarrow$ $\nu+i \epsilon)$, while the lower sign is from [19]-[20].

The result for $\operatorname{Im}_{g}$ was obtained in [19]-[20] by direct calculation of one-loop Feynman diagrams, and so it can be checked only by a similar

6 This cancelation becomes ineffective only at the ends points of the quark phase space where, for instance, one quark carries nearly all the proton momentum, while other ones are wee. The contributions from these regions to the total amplitude are, indeed, suppressed by the Sudakov effects. But in any case, the contributions from these end point regions give only power corrections to the total amplitude, even ignoring the additional Sudakov suppression. 
independent calculation. But there at least one simple argument following from general analyticity properties, in favour of the approach used in [6].

Let us first consider our amplitude $T_{g}\left(\nu, M^{2}\right)$ in the Euclidean region $M^{2}<0$. There is no discontinuity in $M^{2}$ in this region, and the only discontinuity is due to the s-cut. So, $i \epsilon$ terms enter unambiguously into all denominators as: $\nu=(s-u) \rightarrow \nu+i \epsilon$, and the terms $f_{1}$ and $f_{2}$ in Eq.(9) will be multiplied by the same analytic function $g\left(\nu, M^{2}\right)$ :

$$
\left[f_{1}(x, y)+f_{2}(x, y)\right] g\left(\nu+i \epsilon, M^{2}\right) \text {. }
$$

Let us continue now this expression into the Minkowski region $M^{2}>0$. How it may be that after the analytic continuation the functions $f_{1}(x, y)$ and $f_{2}(x, y)$ will be multiplied by two different functions, as in [19]-[20] (see Eq. $(9))$ :

$$
\left[f_{1}(x, y) g\left(\nu+i \epsilon, M^{2}\right)+f_{2}(x, y) g^{\star}\left(\nu+i \epsilon, M^{2}\right)\right] \quad ?
$$

In conclusion, I would like to emphasize that in spite of some "internal" differences between the results from [6]-[7] and [19]-[20], the final curves for $d \sigma / d y$ are practically the same, and Fig. 3 gives a good representation of both answers. So, in any case, the qualitative conclusions agree: under the conditions of the E791 experiment, the process considered appeared to be weakly sensitive to the profile of the pion wave function $\phi_{\pi}(x)$ and, unfortunately, these data can not discriminate between, say, $\phi_{\pi}^{a s y}(x)$ and $\phi_{\pi}^{C Z}(x)$.

\section{Acknowledgements}

I am grateful to the Theory Group of JLab and Organizing Committee and, in particular, to A.V. Radyushkin for a kind hospitality and support. 


\section{References}

[1] E.M. Aitala et. al. (E791 Collaboration), Phys. Rev. Lett. 86 (2001) 4768 ; hep-ex/0010043

D. Ashery, hep-ex/9910024

[2] S.F. King, A. Donnachie and J. Randa, Nucl. Phys. B167 (1980) 98

J. Randa, Phys. Rev. D22 (1980) 1583

[3] G. Bertsch, S.J. Brodsky, A.S. Goldhaber and J.F. Gunion, Phys. Rev. Lett. 47 (1981) 297

[4] L. Frankfurt, G.A. Miller and M. Strikman, Phys. Lett. B304 (1993) 1; Found. of Phys. 30 (2000) 533; hep-ph/9907214; hep-ph/0010297

[5] N.N. Nikolaev, W. Schafer and G. Schwiete, Phys. Rev. D63 (2001) 014020 ; hep-ph/0009038

[6] V. L. Chernyak, Phys. Lett. B516 (2001) 116 ; hep-ph/0103295

[7] V. L. Chernyak and A. G. Grozin, Phys. Lett. B517 (2001) 119 ; hep-ph/0106162

[8] D. Muller, D. Robaschik, B. Geyer, F.-M. Dittes and J. Horejsi, Forts. Phys. 42 (1994) 101

[9] A. V. Radyushkin, Phys. Lett. B385 (1996) 333; Phys. Rev. D56 (1997) 5524

[10] X. Ji, Phys. Rev. Lett. 78 (1997) 610 ; J. Phys. G24 (1998) 1181

[11] V. L. Chernyak and A. R. Zhitnitsky, JETP Lett. 25 (1977) 510 ;

Sov. J. Nucl. Phys. 31 (1980) 544

[12] V. L. Chernyak, V. G. Serbo and A. R. Zhitnitsky, JETP Lett. 26 (1977) 594; Sov. J. Nucl. Phys. 31 (1980) 552

[13] A. V. Efremov and A. V. Radyushkin, Phys. Lett. B94 (1980) 245;

Teor. Math. Phys. 42 (1980) 97

[14] G. P. Lepage and S. J. Brodsky, Phys. Lett. B87 (1979) 359; Phys. Rev. D22 (1980) 2157 
[15] V. L. Chernyak and A. R. Zhitnitsky, Phys. Rep. 112 (1984) 173

[16] M. Gluck, E. Reya and A. Vogt, Eur. Phys. J. C5 (1998) 461

[17] K.J. Golec-Biernat, A.D. Martin and M.G. Ryskin, Phys. Lett. B456 (1999) 232; hep-ph/9903327

[18] V. L. Chernyak and A. R. Zhitnitsky, Nucl. Phys. B201 (1982) 492

[19] V. M. Braun, D. Yu. Ivanov, A. Schafer and L. Szymanowski, Phys. Lett. B509 (2001) 43; hep-ph /0103275

[20] V. M. Braun, D. Yu. Ivanov, A. Schafer and L. Szymanowski, hep-ph/0204191 